\title{
Structural Features of Colonization Factor Antigen I (CFA/I) Pili (Fimbriae) of Enterotoxigenic Escherichia coli
}

\author{
Xiang-Qi Mu ${ }^{1}$, Stephen J. Savarino ${ }^{2}$, Yasemin Akay ${ }^{1}$, Annette McVeigh ${ }^{2}$, \\ Frederick Cassels ${ }^{3}$, Esther Bullitt ${ }^{1}$ \\ ${ }^{1}$ Dept Physiol \& Biophysics, Boston Univ School of Medicine, 715 Albany St, Boston MA 02118 \\ ${ }^{2}$ Naval Medical Research Center and ${ }^{3}$ Walter Reed Army Inst of Research, 503 Robert Grant Ave, \\ Silver Spring, MD 20910
}

Enterotoxigenic Escherichia coli (ETEC) express adhesive pili (also called "fimbriae") that mediate attachment to the small intestine during the earliest pathogenic stages in the host. Colonization factor antigen I (CFA/I) pili are prevalent among ETEC throughout the developing world, archetype of eight genetically related ETEC colonization factors, and generally recognized as both a virulence factor and protective antigen. As such, it has served as a target antigen for ETEC vaccine development, yet few details of its fine architecture are known. To determine the structural features that allow CFA/I pili to operate as bacterial tethers in the intestinal tract, we have examined in detail the structure of $\mathrm{CFA} / \mathrm{I}$ and calculated a three-dimensional reconstruction.

Most CFA/I pili are long helical filaments, $\sim 8 \mathrm{~nm}$ in diameter and, on average, $1 \mu \mathrm{m}$ in length. Additional morphologies observed by transmission electron microscopy include gentle curvature of the filament, sharp bends in the filament, and regions of the filaments that are unwound to a thin fibril approximately $2-3 \mathrm{~nm}$ in diameter.

Results from our 3D reconstruction of CFA/I pili establish that these are helical filaments with a repeat of 19 fimbrial subunits in 6 turns of the helix (3.17 subunits per turn) with a 1.04 nm rise per subunit along the helical axis. Each subunit has a bi-lobed appearance, and is positioned in the filament with its long axis approximately perpendicular to the helical axis. A comparison of our 3D reconstruction of CFA/I pili to those of uropathogenic and respiratory pili elucidates further the specialization required for survival in different microenvironments. We show previous results from P-pili expressed on uropathogenic E. coli and Hib pili from $H$. influenzae to illustrate similarities and differences in structures with the same function, namely adhesion to the human host cell. Both P-pili and CFA/I pili can unwind to fibrillar structures, and show gentle curvature and sharp bends. Subunit-subunit interactions along the helical axis, between the $n^{\text {th }}$ subunit and the $(n+1)$ subunit, are much stronger in P-pili than in CFA/I pili. This difference is even more striking in a comparison with Hib pili, in which the major subunit-subunit interactions are along the helical axis rather than around the filament.

The major structural subunit of CFA/I pili, CfaB, was visualized by immunoelectron microscopy (IEM) of both negatively stained bacteria and of CFA/I pili isolated from wild type ETEC. Our IEM data show that $\mathrm{CfaB}$ is distributed along the length of the fimbrial structure. Increased antibody binding just below the fimbrial tips indicates increased exposure of a highly immunogenic epitope near the distal end of the fimbrial structure. These data confirm that the $3 \mathrm{D}$ reconstruction we have calculated of the CFA/I pili is a reconstruction of the assembled form of the CfaB pilin protein.

Our studies on the structural architecture of CFA/I pili have begun to elucidate strategies by which these bacteria remain attached to the host cell under physiologic conditions designed to thwart infection. It is hoped that such structural knowledge will promote more rational design of interventions to prevent ETEC disease. 


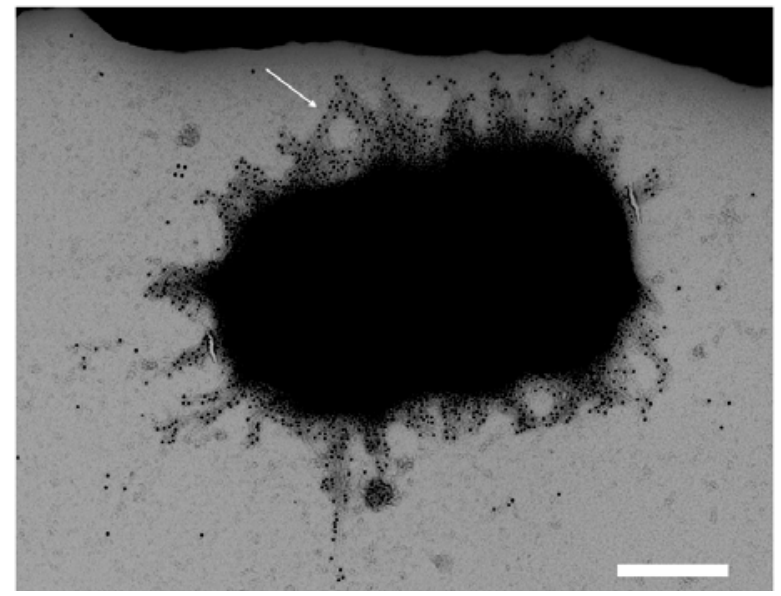

\section{Localizing the major structural pilin, $\mathrm{CfaB}$}

The major structural subunit of CFA/I pili, CfaB, was visualized by immunogold electron microscopy (IEM) of negatively stained whole bacteria expressing CFA/I pili.

IEM data show that $\mathrm{CfaB}$ is distributed along the length of the fimbrial structure (white arrow).

These data confirm that the 3-D reconstruction we have calculated of the CFA/I pili is a reconstruction of the assembled form of the $\mathrm{CfaB}$ pilin protein. Magnification bar, 0.5 microns.

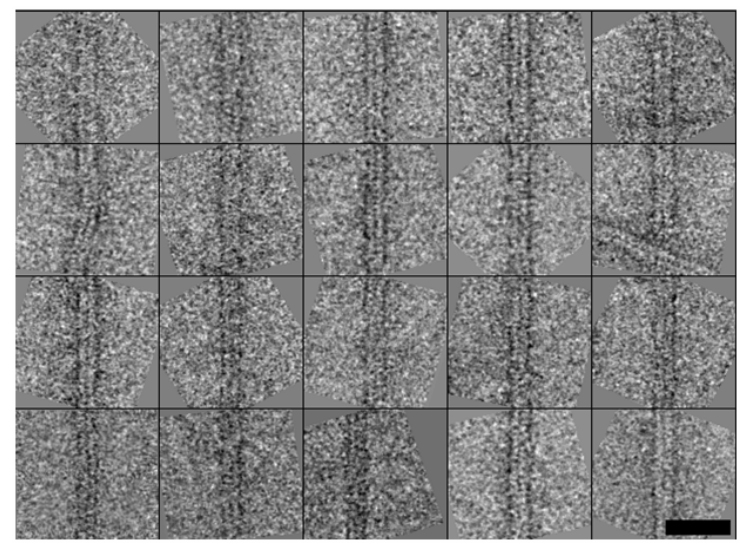

Examples of CFA/I filament segments boxed as individual particles for 3-D reconstruction

Small, straight regions of CFA/I pili are boxed, and treated as single particles to begin the image analysis procedure. After rotational alignment (as shown to the left) each particle is tested to determine whether its quality is sufficient for inclusion in further image processing. For example, in Row 2, Columns 1 and 5 were discarded, due to a bend in the filament and a crossing filament, respectively. Magnification bar $250 \AA$.

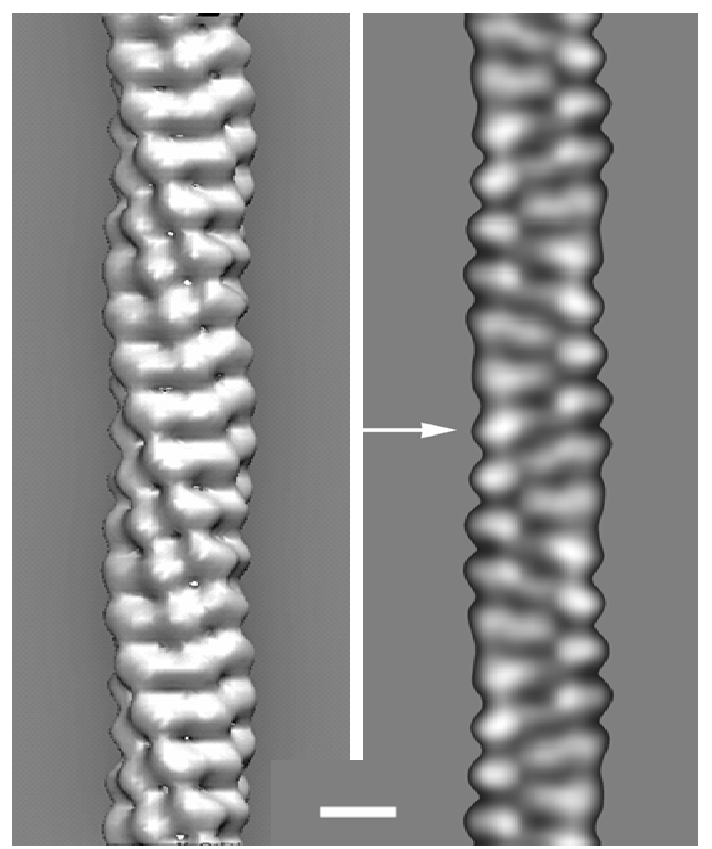

\section{3-D Reconstruction of CFA/I pili}

After image processing, a back projection of the electron microscopy data is computed, providing a 3-D reconstruction of CFA/I pili.

Results from our 3-D reconstruction of CFA/I pili (left) establish that these are helical filaments $\sim 8 \mathrm{~nm}$ in diameter, with a repeat of 19 subunits in 6 turns of the helix (3.17 subunits per turn). There is a $10.4 \AA$ $(=1.04 \mathrm{~nm})$ rise per subunit along the helical axis, and a rotation between subunits of $113.3^{\circ}$ around the helical axis.

A surface view of the reconstruction (far left) shows a left-handed long pitch helix, with bi-lobed subunits oriented approximately horizontally (perpendicular to the helical axis).

A projection map of the CFA/I pilus reconstruction (near left) mimics the data visible in electron microscope images; compare this result to the montage of CFA/I pili segments. Mag bar, $50 \AA$. 